\section{Erstmalig prospektiv nachgewiesen:}

\section{Eisenchelat-Therapie verlängert das Gesamtüberleben deutlich bei transfusionspflichtigen MDS- Patienten}

Im Rahmen des Annual Meeting of the American Society of Hematology (ASH) in Atlanta, GA, USA, im Dezember 2007 wurden neue Daten vorgestellt, die die Wirksamkeit von Deferasirox nochmals eindrucksvoll belegen. Im Mittelpunkt steht die prospektive Studie von Rose et al. [1], einer unabhängigen französischen Arbeitsgruppe, die für die Chelat-Therapie erstmalig einen Vorteil im Hinblick auf das Gesamtüberleben von transfusionspflichtigen MDS-Patienten zeigen konnten.

In der longitudinalen, prospektiven, multizentrischen Studie wurden die hämatologischen Daten, die Anzahl an Erythrozytenkonzentrat
(EK)-Transfusionen und der Chelationsstatus (mit oder ohne Eisenchelat-Therapie) von 170 MDS-Patienten aus insgesamt 18 französischen GFM-Zentren (Groupe Francophone des Myélodysplasies) erfasst und ausgewertet. Die Patienten erhielten alle innerhalb eines Monats das erste EK, nach 2 Jahren erfolgte die Follow-upUntersuchung. $46 \%$ der Probanden erhielten über mindestens 6 Monate eine EisenchelatTherapie (Deferoxamin, Deferiprone oder Deferasirox). Die mediane Überlebensdauer bei Patienten unter Eisenchelat-Therapie wurde mit 115 Monaten angegeben, bei Patienten, die keine Eisenchelat-Therapie erhielten, betrug 51 Monate $(\mathrm{p}<0.0001)$. Damit weisen chelierte im Vergleich zu nicht-chelierten Patienten einen signifikanten Überlebensvorteil von 64 Monaten, also von mehr als 5 Jahren auf.

Auch nach Bereinigung statistischer Faktoren (Geschlecht, Alter, IPSS (International Progno- stic Scoring System), Transfusionsstatus) blieb der Überlebensvorteil signifikant.

Die auf dem ASH-Kongress vorgestellten Studien unterstreichen bereits bekannte positive Ergebnisse zur Wirksamkeit von Deferasirox (Exja$\left.\mathrm{de}^{\circledR}\right)$ : Eisenchelat-Therapie bietet chronisch transfundierten MDS-Patienten einen Überlebensvorteil, insbesondere bei einem niedrigen oder mittleren Risiko nach der IPSS-Klassifizierung.

\section{Quellen}

1 Rose C, Brechiniac S, Vassilief D et al.: Positive impact of iron chelation therapy (CT) on survival in regularly transfused MDS patients. A prospective analysis by the GFM. Blood 2007;110 (11):Abstract 249

Weitere Informationen bei

Novartis Pharma GmbH

Dr. Irene Roth

Roonstraße 25, 90429 Nürnberg

Irene.roth@novartis.com

\section{EU-Zulassung für CAELYX ${ }^{\circledR}$ in Kombination mit Bortezomib beim multiplen Myelom}

Die Europäische Kommission hat auf Empfehlung des European Committee for Medicinal Products for Human Use (CHMP) am 14. Dezember 2007 die Zulassung für ein neues Anwendungsgebiet von CAELYX ${ }^{\circledR}$ erteilt. Demzufolge ist CAELYX ${ }^{\circledR}$ in Kombination mit Bortezomib nun auch zugelassen zur Behandlung des progressiven multiplen Myeloms bei Patienten, die zumindest eine vorangegangene Therapie erhalten haben und die sich bereits einer Knochenmarktransplantation unterzogen haben bzw. dafür ungeeignet sind [1]

Die Zulassungserweiterung basiert auf den Daten einer internationalen Phase-III-Studie, in der die Kombination von pegyliert liposomalem Doxorubicin (PLD) und Bortezomib mit einer Bortezomib-Monotherapie bei 646 Patienten mit rezidiviertem oder therapierefrak- tärem multiplen Myelom verglichen wurde [2, 3]. Die Auswertung nach einer medianen Nachbeobachtungsdauer von 14 Monaten zeigte, dass die Patienten von der Kombinationstherapie mit CAELYX ${ }^{\circledR}$ eindeutig profitierten: Die Kombinationstherapie führte im Vergleich zur Bortezomib-Monotherapie zu einer signifikanten Verlängerung der medianen Dauer bis zur Progression von 6,5 auf 9,3 Monate $(p=0,000004)[2]$. Zudem kam es zu einer signifikanten Verbesserung der Gesamtüberlebensrate ( 82 vs. $75 \%$; p < 0,05) und der Ansprechrate (52 vs. 44\%; $\mathrm{p}=0,050$ ) bei den Patienten, die mit Bortezomib und PLD behandelt wurden [2]

Das Sicherheitsprofil der Kombinationstherapie war vorhersehbar und die Nebenwirkungen beherrschbar, wobei es sich als konsistent mit den für beide Substanzen bekannten Toxizitäten erwies [2]. Unerwünschte Ereignisse Grad 3 oder 4 traten im Kombinationstherapiearm häufiger auf und waren durch eine Zunah- me der Myelosuppression und gastrointestinaler Toxizitäten verursacht. Es wurde ein erhöhtes Risiko für Neutropenie, Thrombozytopenie, Diarrhö, Erbrechen, Asthenie, Fatigue und palmoplantare Erythrodysästhesie beobachtet, während weder eine Zunahme von Neuropathien noch von kardialen Ereignissen zu verzeichnen war. Thromboembolische Ereignisse waren ebenfalls selten, so dass keine Thromboseprophylaxe erforderlich ist [2].

\section{Quellen}

1 Fachinformation CAELYX®, Stand: Dezember 2007 2 Harousseau JL, et al. ASCO 2007 Abstract \# 8002 bzw. mündliche Präsentation (siehe www.asco.org)

3 Orlowski RZ, et al. J Clin Oncol 2007; 25: 3892-3901

Weitere Informationen bei

Medizin \& Markt GmbH

Franziska Wagenschwanz

Schackstraße 1, 80539 München

Tel. +4989383930-22

wagenschwanz@medizin-und-markt.de

\title{
Ticker+++ Ticker+++ Ticker+++ Ticker+++ Ticker+++ Ticker+++ Ticker+++
}

Bayer Vital GmbH. Seit Februar 2008 steht Patienten mit Hämophilie A die neue Packungsgröße KOGENATE ${ }^{\circledR}$ Bayer 2000 I.E. zur Verfügung. Damit vereinfacht Bayer Vital für viele Hämophiliepatienten die Verabreichung des rekombinanten Faktor VIII, die sich regelmäßig den in ihrem Blut fehlenden Gerinnungsfaktor VIII zuführen müssen. Für jeden Faktor-VIII-Bedarf sowohl in der Prophylaxe als auch in der Bedarfstherapie steht nun eine passende Größe zur Verfügung.

Weitere Informationen bei

Bayer Vital GmbH

Dr. Michael Diehl

michael.diehl@bayerhealthcare.com
Janssen-Cilag GmbH. Mit der Einführung des Proteasom-Inhibitors Bortezomib (Velcade ${ }^{\circledR}$ ) haben sich in der Vergangenheit die therapeutischen Möglichkeiten beim rezidivierten Myelom deutlich erweitert: Der Wirkstoff ermöglicht eine hohe Ansprechqualität und ist langfristig von Nutzen, wie auch eine aktuell auf der Jahrestagung der American Society of Hematology (ASH) vorgestellte Folgeauswertung der CRESTStudie bestätigt.

Weitere Informationen bei

SanCom Creative Communication Solutions $\mathrm{GmbH}$ Yasmin König

koenig@sancom-ccs.de
Amgen GmbH. Pegfilgrastim (Neulasta ${ }^{\circledR}$ ) ist ein kovalentes Konjugat des rekombinanten humanen G-CSF mit einem PEG-Molekül von 20 kD. Dadurch ist die renale Clearence vermindert und die Verweildauer im Organismus verlängert. Aufgrund der längeren Halbwertszeit ist nur eine jeweils einmalige Injektion von $6 \mathrm{mg}$ nach einem Chemotherapiezyklus ausreichend, um eine Neutropenie oder eine durch Neutropenie verursachte Infektion zu verhindern.

Weitere Informationen bei

POMME-med GmbH

Dr. rer. nat. Petra Ortner

ortner@pomme-med.de 